\title{
The Effect of Autonomous Vehicles on Traffic
}

\author{
Bernhard Friedrich
}

\subsection{Introduction}

\section{Objective}

Autonomous vehicles maneuver in traffic through road networks without requiring humans as supervisors or decision makers. Autonomous vehicles increase comfort for their passengers by removing the need for them to perform driving tasks. Autonomous vehicles provide new mobility opportunities for groups of people that thus far have been partially or entirely excluded from participation in public life due to mobility restrictions.

In addition to the benefits that autonomous vehicles potentially provide their users, the social benefits that would come with their proliferation are of interest. For it is clear that autonomous driving does not lead to a loss of safety or efficiency of road transport but rather improves them. This paper considers the traffic impact of autonomous vehicles, looking specifically at the efficiency of using the existing infrastructure.

The efficiency of the transport infrastructure is determined by its capacity. On highways, the capacity is dependent to a large degree on the maximum possible flow of traffic on the road sections as well as the capacity of entry, merging and exit lanes at grade-separated traffic intersections. In the city road network, and on country roads with through-roads, the capacities at the intersections are the crucial factor and therefore mostly dependent on traffic signaling. While the capacity at traffic lights is determined by the amount of time required by individual vehicles in the departing lane to pass the node, the capacity of highway sections is determined by the instability that occurs at high traffic volumes and leads to congestion.

\section{B. Friedrich $(\bowtie)$}

Institute of Transportation and Urban Engineering,

Technische Universität Braunschweig, 38092 Braunschweig, Germany

e-mail: friedrich@tu-braunschweig.de 
In order to understand the various factors that determine the effect of traffic on autonomous vehicles, the key characteristics of traffic flow and their interdependent elements are the initial focus of this paper. Building on these foundations, we will derive the impact of autonomous vehicles on the capacity of highway segments as well as on intersections with traffic signals. The impact of autonomous vehicles on the connection quality of journeys covering different infrastructure elements cannot be adequately described in this model. Nevertheless, these considerations provide a preliminary assessment of the potential for optimizing the efficiency of traffic flow that potentially includes autonomous vehicles.

\subsection{Characteristics of Traffic Flow}

\subsubsection{Parameters of Traffic Flow}

In order to develop a mathematical model of traffic flow, we use an abstraction of the road network, the vehicles, the drivers and their behavior. Certain simplifying assumptions are therefore made.

The road network is divided, for example, into road segments and intersections. We investigate here, therefore, either road segments or intersections where consistent conditions, i.e., flatness, sufficient visibility, dry surface, etc. are assumed. With regard to drivers and vehicles, it is expected, among other things, that properties such as reaction time, willingness to engage in risks and technical proficiency follow an empirically proven statistical distribution.

A distinction is to be made between several ways of describing traffic flow. The microscopic model describes the relevant characteristics of a single vehicle $i$ :

- temporal headway $t_{i}(\mathrm{~s})$

- spatial separation $x_{i}(\mathrm{~m})$

- speed $v_{i}(\mathrm{~km} / \mathrm{h})$.

The macroscopic descriptive model takes into consideration many vehicles and the relevant properties of a traffic flow:

- traffic volume $q(\mathrm{veh} / \mathrm{h})$

- traffic density $k(\mathrm{veh} / \mathrm{km})$

- mean speed $v(\mathrm{~km} / \mathrm{h})$.

The traffic flow can be recorded by measuring the parameters of a particular cross-section over a time interval $\mathrm{d} t$ by means of so-called local observations or measurements at a given time over a path interval $\mathrm{d} x$, which are so-called momentary observations (Fig. 16.1). 


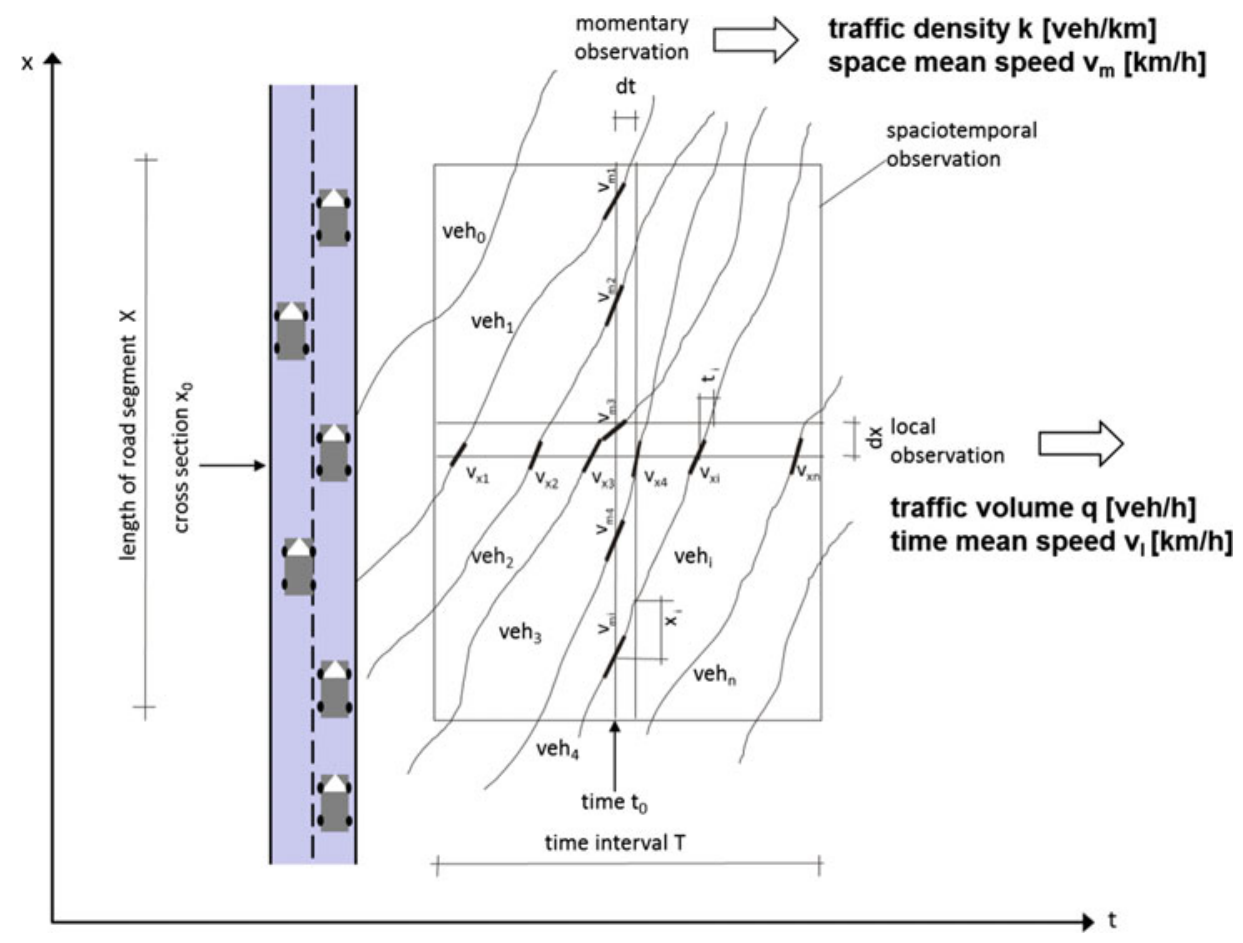

Fig. 16.1 A system of local and momentary measurements. Different traffic speeds occur for the individual speeds recorded locally or momentarily

\subsubsection{Traffic Flow Theory}

Neither the macroscopic parameters $v, q$, and $k$, nor the corresponding microscopic values, define in themselves a traffic state. In order to define a traffic state, knowledge of their interdependencies is a prerequisite. The three macroscopic quantities, traffic volume, traffic density and momentary speed, are dependent on one another according to the equation

$$
q=k \cdot v(k)
$$

Measurements of traffic volume and mean speed resulted in a detectable decrease in speed when traffic volume increases, i.e., with increasing mutual influence of vehicles.

One of the first models to describe traffic flow on an open stretch road came from observations made by Greenshields [1], who researched the relationship between the speed $v$ and the traffic density $k$. With the help of regression analysis, he established a linear relationship for $v=v(k)$ 


$$
v(k)=v_{\mathrm{f}}-v_{\mathrm{f}} / k_{\max } \cdot k=v_{\mathrm{f}} \cdot\left(1-k / k_{\max }\right)
$$

where $v_{\mathrm{f}}$ represents the free flow speed and $k_{\max }$ the maximum traffic density.

Inserted into the equation $q=v \cdot k$, this results in a parabolic relationship between traffic volume and traffic density in the form:

$$
q(k)=v_{\mathrm{f}} \cdot\left(k-k^{2} / k_{\max }\right)
$$

Equations with these parameters are referred to as equations of state and their graphical representations are called fundamental diagrams of traffic flow.

\subsubsection{Model for Stationary Traffic Conditions-Fundamental Diagram}

The fundamental diagram is a graphical illustration of the equation of state for traffic, i.e., the functional relationship between the parameters of traffic volume $q$, traffic density $k$, and the mean momentary, i.e., section-related speed $v$, and represents a curve in three-dimensional space. The orthogonal projections of the curve onto the planes, each spanned by two parameters, result in the familiar fundamental diagram shown in Fig. 16.2. The resulting three diagrams enable a variety of information about the characteristics of traffic flow over a cross section to be depicted and are referred to as the $q$ $v$ diagram, the $q-k$ diagram, and the $k-v$ diagram.

The fundamental diagram shows that, for the same traffic volume $q_{l}$, two different qualities of traffic flow can occur. The threshold $q_{\max }$ separates for $q_{i}<q_{\max }$ the range of high speeds at low traffic densities, i.e., the free and stable flow of traffic, from the range with relatively low speeds and high traffic densities, i.e., the range of unstable and interrupted traffic flow. Empirical studies reveal that the transition between a stable and an unstable traffic state does not run continuously as shown in Fig. 16.2 in idealized form. Rather, in case of high traffic load and triggered by disturbances a transition from the stable to the unstable range takes place. This transition is associated with a significant drop in the traffic volume (Fig. 16.3). In light of these considerations, May and Keller [2] characterized three forms of traffic that occur:

- Free traffic at high speeds and low traffic volumes and densities

- Partially constricted traffic, up to the range of maximum traffic volumes, optimal speed and traffic density

- Constricted traffic with high traffic densities, low traffic volumes and speeds. 


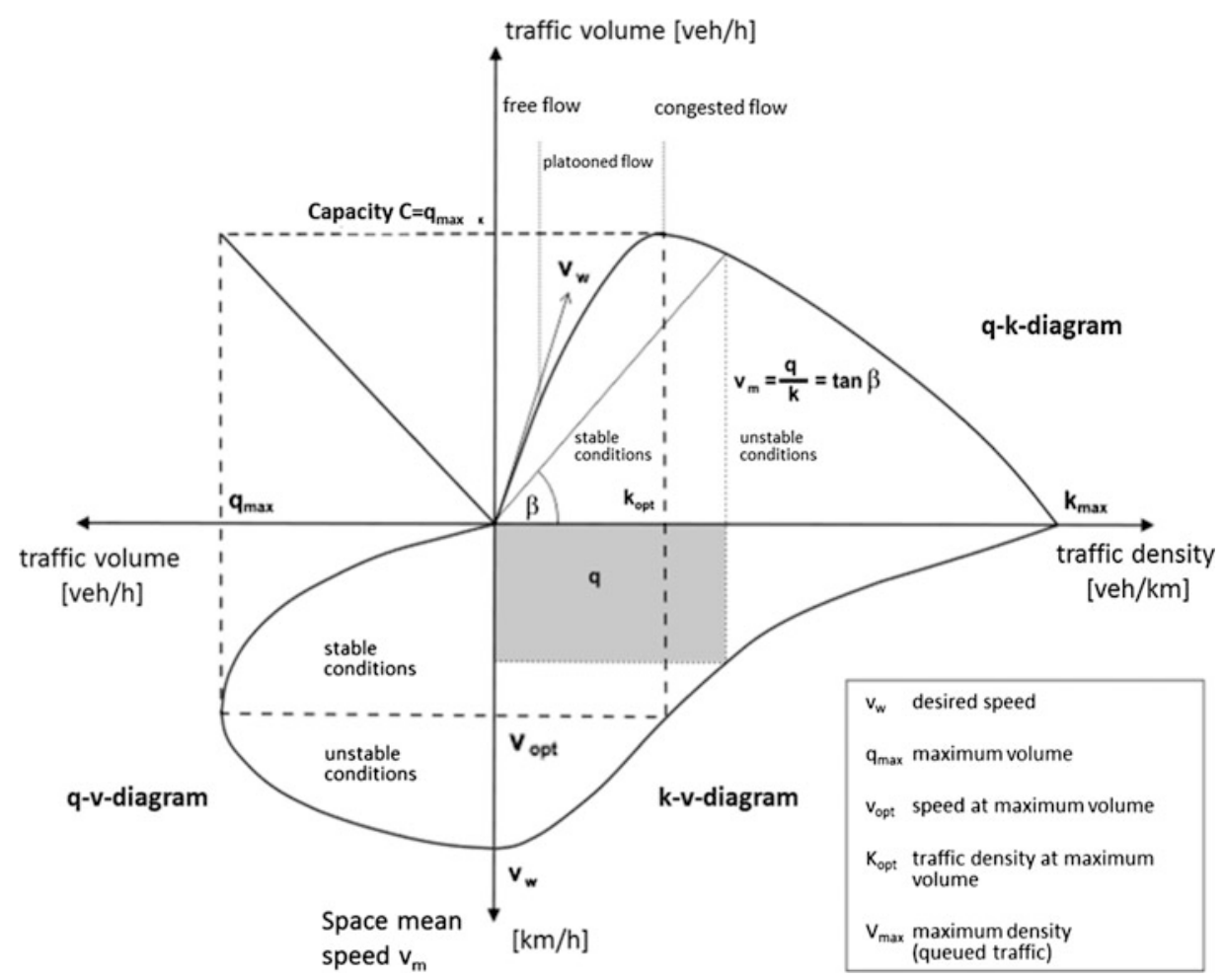

Fig. 16.2 Views of the fundamental diagram according to [3]. Source Handbuch für die Bemessung von Straßenverkehrsanlagen (HBS), S. 3-19, FGSV 2001

Fig. 16.3 Fundamental diagrams with separate ranges for stable and unstable traffic for single- and two-lane roads, according to [2]

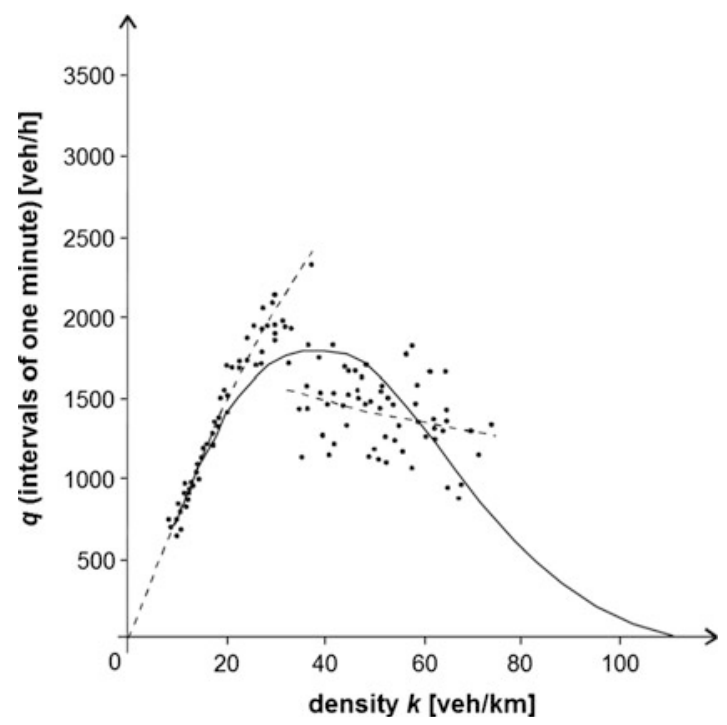




\subsubsection{Capacity and Stability}

The efficiency of the traffic system depends on the capacity of the traffic infrastructure. This capacity is defined as the "largest volume of traffic that a traffic flow can reach at a given distance and traffic conditions at the cross-section determined for this flow" [3]. The capacity is determined by the density of the platoon of vehicles and the speed with which the platoon passes through the cross-section.

Traffic density is determined by the distances between vehicles. The rule of thumb is that the safe distance in meters before the vehicle ahead that a driver should adhere to is half the value of the current speed in kilometers per hour. This well-known rule of "half speedometer distance" is based on a reaction time of less than $1.8 \mathrm{~s}$, since at this value and constant speed, precisely the distance to the preceding vehicle is travelled. This minimum distance is also usually required by law (see, e.g., [4]). For trucks, road regulations explicitly stipulate that, at speeds above $50 \mathrm{~km} / \mathrm{h}$, a minimum travel distance of $50 \mathrm{~m}$ must be maintained, which requires a time interval of $2.25 \mathrm{~s}$ at the maximum speed limit permissible for vehicles over 7.5 tons on highways.

Assuming a reaction time of $1.8 \mathrm{~s}$, the capacity of a lane can be estimated, in a simplified model, at about 2000 vehicles per hour. This applies equally to city streets as to country roads and highways. However, empirical studies show that the headways are on average significantly shorter than $1.8 \mathrm{~s}$ and especially at high traffic volumes amount to $1.0 \mathrm{~s}$. The $15 \%$ percentile of the distribution in these cases is even below $0.5 \mathrm{~s}$ (see also Fig. 15.3 in the chapter by Peter Wagner in this book [5]). This means that $15 \%$ of the vehicles follow a preceding vehicle with a headway of less than 0.5 s. Figure 16.4 shows the corresponding headway distributions for different traffic volume ranges and different speed limits.

Due to the short following distances at relatively high speeds, empirical studies also investigate capacities that may be significantly higher than the stated 2000 veh./h. Furthermore, these studies indicate that there is no exact value where traffic flow stops being stable and breaks down if this value is exceeded. Rather, it can be observed that the capacity is a random parameter that can be represented by a distribution (Fig. 16.5). Investigations of many sections of road [6] show that the capacity of highways are typically Weibull-distributed and, for example for 3-lane highways, show a standard deviation of about $600 \mathrm{veh} . / \mathrm{h}$ (measured in 5-min intervals) and thus an unexpectedly wide variability.

The expected value of the capacity corresponds in this stochastic depiction to a nominal capacity and represents the 50th percentile of that traffic volume amount that was the starting point of a breakdown in traffic flow. The closer the traffic load is to, or the further it lies above, this nominal capacity, the higher the probability of traffic breakdown and traffic congestion.

Traffic breakdown results in all cases, passing through a transient state of synchronized traffic flow to congested traffic. Traffic recovery occurs also via a transient state of synchronized traffic flow, back to a steady flow of traffic with higher speeds (Fig. 16.6). 


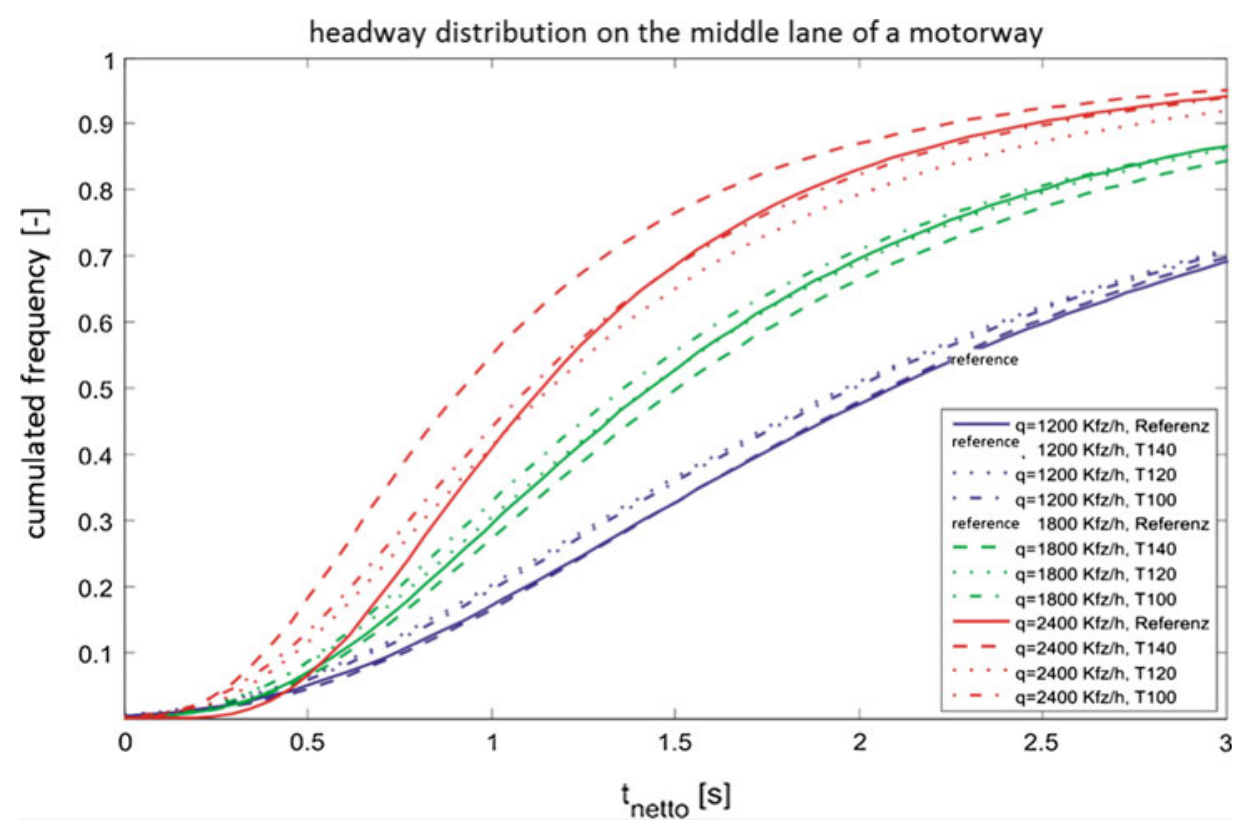

Fig. 16.4 Distribution functions of the time gap distribution for different traffic loads and speed restrictions [7]

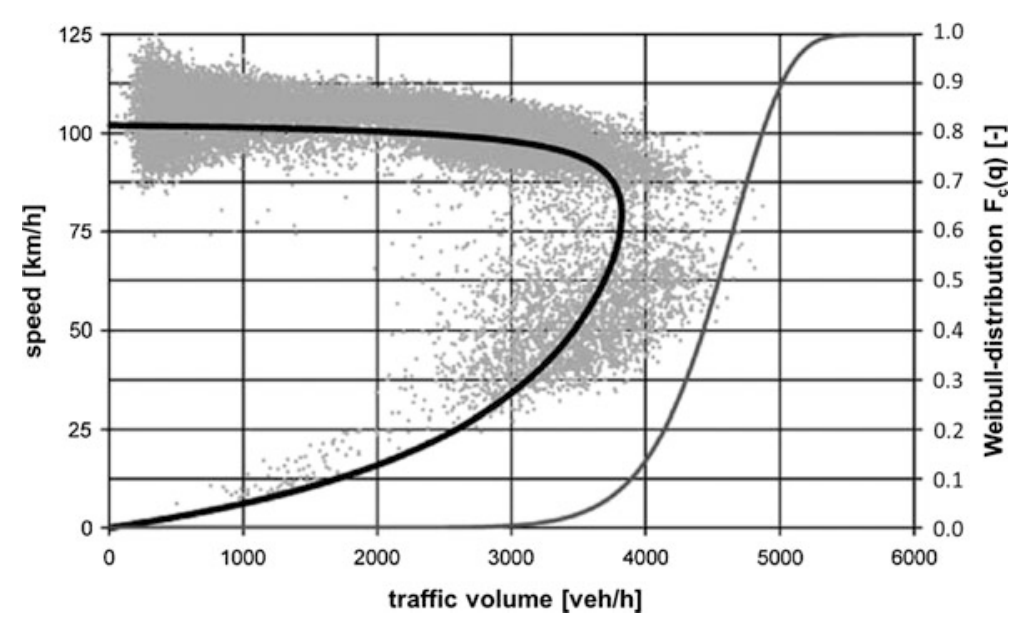

Fig. 16.5 Values for 5-min intervals in the qv graph and the related capacity distribution for a cross section of a two-lane highway, according to [6]

The traffic volume also decreases in the transitions to synchronized or to congested traffic and a smaller recovery takes place. This effect of a "capacity drop" is caused by the fact that drivers, in keeping a greater distance when leaving the downstream traffic-jam front, 


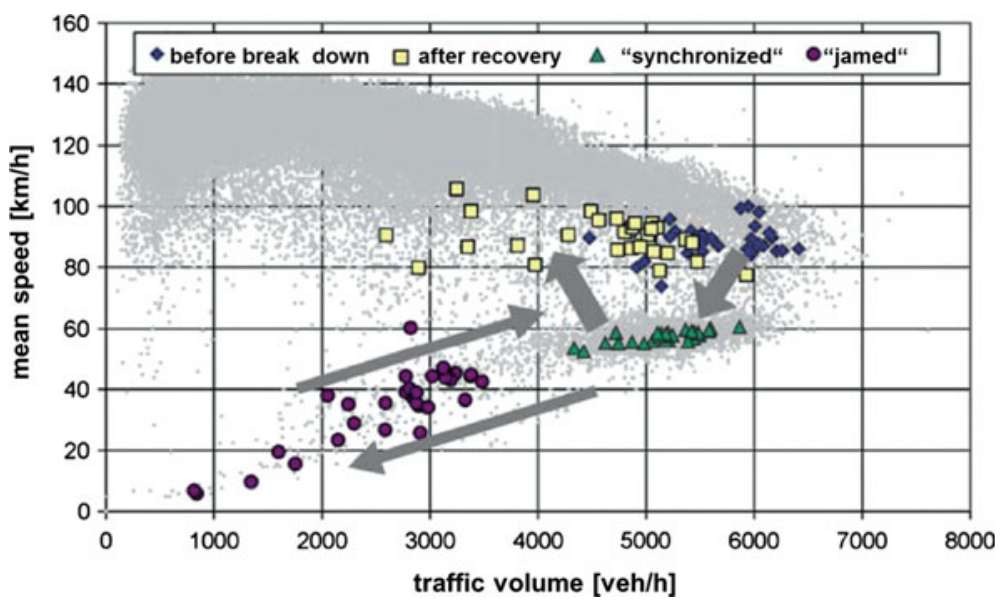

Fig. 16.6 Pattern of traffic dynamics with the transitions from the state of stable traffic into the states of synchronized and congested traffic. The values were measured on a three-lane highway in 5-min intervals, according to [6]

maintain a greater distance than before in flowing traffic before the breakdown in traffic flow.

According to [8], this capacity drop is 5-6\%; studies on German highways reported values between 4 and $12 \%$ [6].

\subsection{The Effect of Autonomous Vehicles on Traffic}

The efficiency of the transport system depends on the capacity of a transport infrastructure. When a transport infrastructure is used by autonomously driving vehicles, the capacity will differ from that of a transport infrastructure used by human drivers. Either the capacity of the route sections of the intersections or define the traffic flow. While the capacities of the intersections and the traffic signals are relevant to the performance of urban road networks, on highways the capacities of open stretches of road are of principal importance. For this reason, the following analysis considers the capacity for both cases, taking into account that a yet-unknown proportion of vehicles drives autonomously.

\subsubsection{Sections of Highways}

\subsubsection{Capacity}

The capacity of a traffic lane is determined by the maximum number of vehicles that can pass through a cross section per unit of time. It is determined by the density of the vehicle platoon and the speed with which the platoon passes through the cross-section. The 
equation of state that describes the relationship between these fundamental characteristics of traffic flow is:

$$
q=k \cdot v(k)
$$

In a homogeneous traffic flow, the density is easily determined and results from the reciprocal of the footprint of a vehicle [9]:

$$
k=\frac{1}{v T_{h}+L} .
$$

In this context, $T_{h}$ is the temporal distance (time gap) to the preceding vehicle and $L$ is the length of a vehicle. Since the capacity represents the maximum traffic volume $q_{\max }$, this is consequently a function of $v, T_{h}$, and $L$. If only human drivers control the vehicle, the capacity $C_{h}$ results, with:

$$
C_{h}=q_{\max }=\frac{v}{v T_{h}+L} .
$$

Analogously, the capacity $C_{a}$ is described in a traffic flow that purely consists of autonomous vehicles by the following function, where $T_{a}$ represents the time gap preferred by autonomous vehicles.

$$
C_{a}=\frac{v}{v T_{a}+L}
$$

The ratio of the two capacity values and hence the change in the capacity is determined by the relation

$$
C_{a} C_{h}=\left(v T_{h}+L\right) /\left(v T_{a}+L\right) .
$$

In order to evaluate the effect of autonomous vehicles on capacity values, values are used for the parameters of the capacity formula that are empirically demonstrated for today's conditions. So it seems reasonable to assume as the mean speed at which the capacity is reached the value $v=80 \mathrm{~km} / \mathrm{h}(22 . \overline{2} \mathrm{~m} / \mathrm{s})$. For the footprint of an average passenger car, the broadly accepted mean vehicle length is $4.5 \mathrm{~m}$ and the minimum safety distance to the vehicle ahead is $3.0 \mathrm{~m}$, thus $L_{\mathrm{car}}=7.5 \mathrm{~m}$ is used. The mean length of a truck is estimated to be $18 \mathrm{~m}$, which is the weighted average of the lengths of a truck $(18.75 \mathrm{~m})$ and of a semi-trailer $(16.50 \mathrm{~m})$. For the footprint of a truck, assuming that a $3.0 \mathrm{~m}$ distance is kept before the vehicle ahead, $L_{\text {truck }}=21 \mathrm{~m}$. As a reasonable value for the mean following distance at high traffic volumes, the empirical studies provide a value of $T_{h}=1.15 \mathrm{~s}$.

For the change in capacity for autonomous driving, the changed time gap $T_{a}$ for the following vehicle is the deciding factor. A technically feasible and, at the same time, 


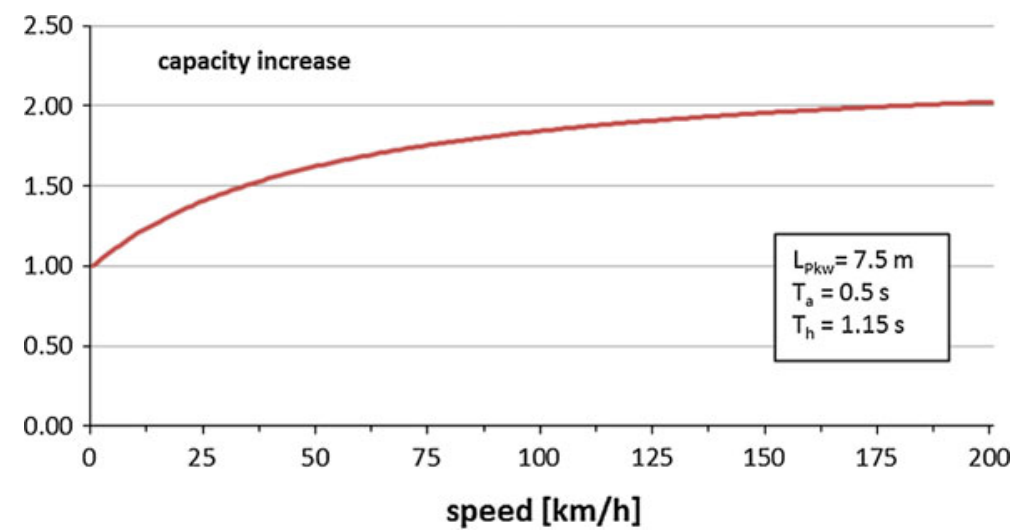

Fig. 16.7 Capacity increase that, depending on the speed, would result in an exclusively autonomous vehicle fleet (only passenger cars)

acceptable value from the perspective of road users appears to be $T_{a}=0.5 \mathrm{~s}$. Depending on the traffic conditions, this very short following distance already occurs in up to $20 \%$ of all following distances. This distance therefore seems acceptable provided that safety is ensured from a technical perspective.

For the assumed values, the capacity and thus the maximum flow rate in the case of purely autonomous traffic would significantly increase using the formulas derived above (factor 1.78) (Fig. 16.7).

Compared to today's observed capacity values of a lane of $2200 \mathrm{veh} . / \mathrm{h}$, an increase of traffic volume to about $3900 \mathrm{veh}$./h would thus be possible with purely autonomous traffic.

If heavy traffic is included in the traffic flow, the mean footprint of the vehicles can be deduced from a sum that is weighted with the proportion of heavy traffic $\omega$. The traffic density is obtained in turn from the reciprocal of the mean footprint of a vehicle with

$$
k=\frac{1}{(1-\omega)\left(v T_{h}+L_{\mathrm{Pkw}}\right)+\omega\left(v T_{h}+L_{\mathrm{Lkw}}\right)} .
$$

For this capacity, the following correlation arises

$$
\mathrm{C}=\frac{v}{(1-\omega)\left(v T_{a}+L_{\mathrm{Pkw}}\right)+\omega\left(v T_{a}+L_{\mathrm{Lkw}}\right)}
$$

If one assumes a moderate speed of $80 \mathrm{~km} / \mathrm{h}$ for autonomous traffic on German motorways, we obtain the functional relationship shown in the following Fig. 16.8. If trucks make up $15 \%$ of traffic, which is typical on German motorways, a capacity of about 3877 veh./h would be achieved, which is almost twice the value compared to today's empirically proven capacity. If, for the sake of a plausibility check and with otherwise unchanged parameters, one inserts $T_{a}=1.15 \mathrm{~s}$ into the capacity formula, this 


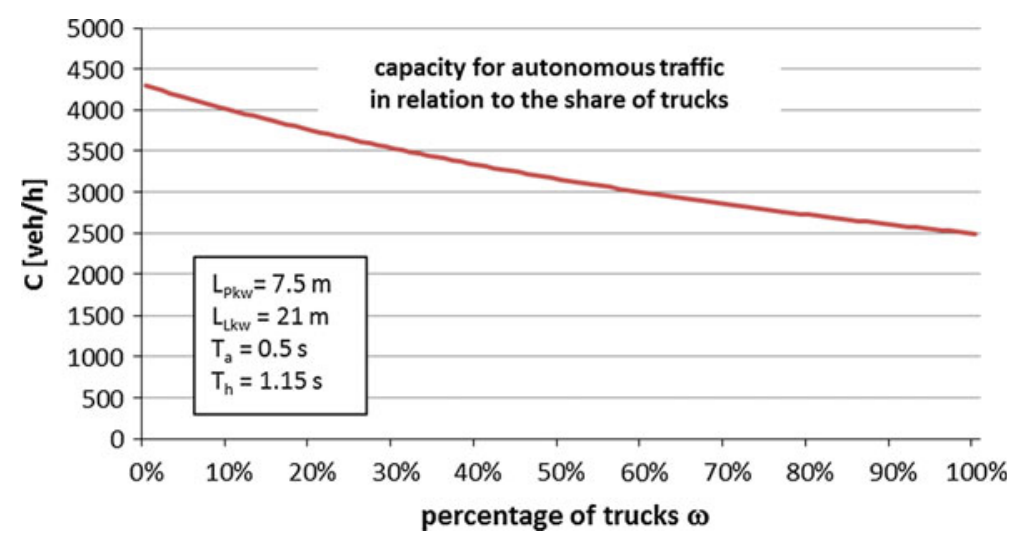

Fig. 16.8 Capacity of a lane in purely autonomous traffic in relation to the share of trucks

results in a capacity of about $2280 \mathrm{veh} . / \mathrm{h}$ for a heavy traffic share of $15 \%$. This value corresponds to the measured capacity at present conditions and confirms the right choice of the computational approach as well as the parameters.

In mixed traffic, where autonomous vehicles are represented by a share of $\eta$ in the total volume, the capacity $C_{m}$ is additionally dependent on percentage $\eta$ :

$$
\mathrm{C}_{m}=\frac{v}{\eta v T_{a}+(1-\eta) v T_{h}+L_{\mathrm{Pkw}}} .
$$

If one inserts realistic values into the equation here, again with $v=80 \mathrm{~km} / \mathrm{h}$ and $L_{\text {car }}=7.5 \mathrm{~m}$, the correlation shown in the following graph results (Fig. 16.9). From the graph, it is clear that the capacity increases more slowly at lower numbers of autonomous vehicles. At $\eta=0.5$ capacity only reaches a value of about 3100 cars $/ \mathrm{h}$, and thus $36 \%$ of the increase that would be possible if all vehicles were autonomous.

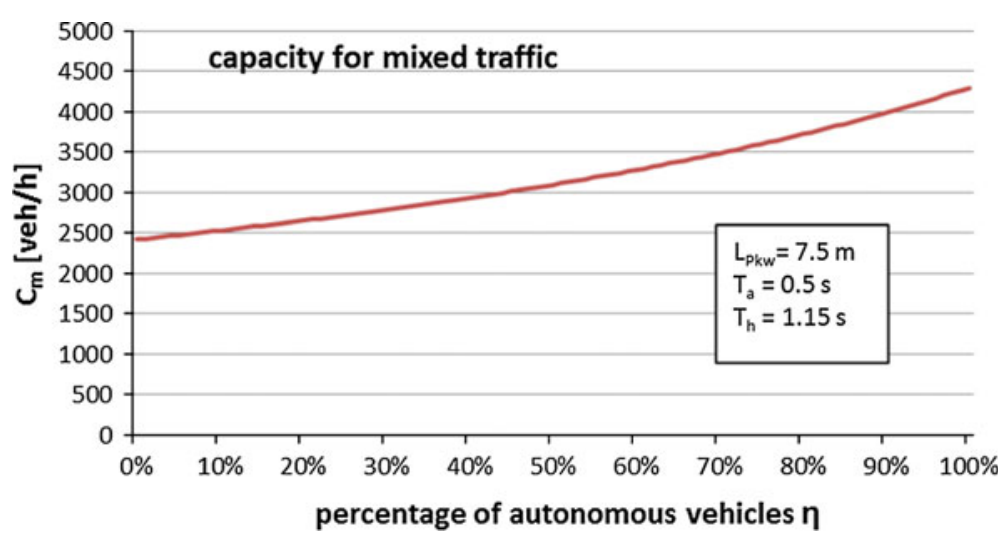

Fig. 16.9 Capacity of a lane in proportion to the share of autonomous vehicles for pure passenger car traffic 
If one also takes into account that autonomous vehicles should allow an additional distance to a vehicle steered by a human driver so as not to harass these drivers, it is slightly more complicated to determine the capacity. In this analysis, the combinations of successive vehicles (a-a, a-h, h-a, and h-h) and the corresponding time gaps $\left(T_{a a}, T_{a h}, T_{h x}\right)$ must be considered in order to arrive at a modified capacity equation:

$$
C_{m}=\frac{v}{\eta^{2} v T_{a a}+\eta(1-\eta) v T_{a h}+(1-\eta) v T_{h x}+L} .
$$

As realistic values for the headways, the values $T_{a a}=0.5 \mathrm{~s}, T_{a h}=0.9 \mathrm{~s}, T_{h x}=1.15 \mathrm{~s}$ can be used. In this analysis, the capacity increases in proportion to the share of autonomous vehicles a little more slowly in the lower range and reaches for $\eta=0.5$ a value of $2850 \mathrm{veh} . / \mathrm{h}$ on the way to achieving a capacity value of almost $4300 \mathrm{veh} . / \mathrm{h}$ where $100 \%$ of vehicles are autonomous (Fig. 16.10).

The same procedure can be used to estimate the capacity for pure heavy-vehicle traffic that could be organized on a single lane of a highway. Not changing our assumptions for the required time gaps, a required space of $L=21 \mathrm{~m}$ is again assumed. For purely autonomous driving, these input values result in a capacity value of 2420 trucks/h, compared to a capacity value of 1720 trucks/h as achievable for human drivers.

\subsubsection{Stability}

Besides the capacity that equates to the greatest traffic volume that a traffic flow at given road and traffic conditions at a cross-section can achieve, the stability of the traffic flow is an important factor in its efficiency. This becomes clear when the capacity is considered as a stochastic variable that represents the probability of traffic breakdown as a function of traffic intensity. The greater the standard deviation of the probability distribution, the greater is the likelihood of traffic breakdown at lower traffic volumes and thus instability.

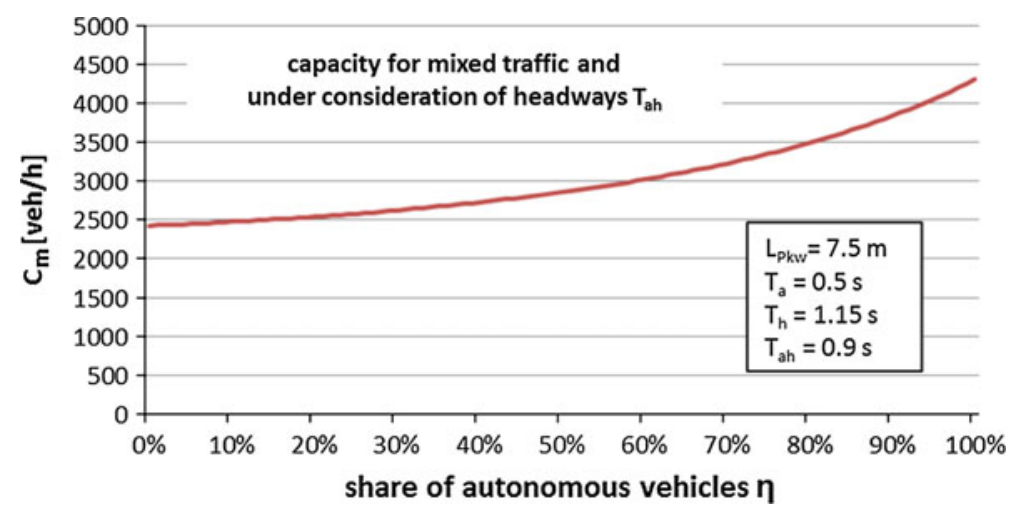

Fig. 16.10 Capacity of a lane in relation to the share of autonomous vehicles for pure car traffic, taking into account larger time gaps for autonomous vehicles following vehicles driven by people 
If breakdown occurs, capacity is reduced by the effect of "capacity drop" noticeable at the magnitude given in the above literature-by about $10 \%$. Given the same number of lanes and same traffic framework conditions (traffic volume, proportion of heavy traffic), different spatial and temporal factors result in different capacity distribution functions. The key factors in this regard are the speed and the time gap distributions. The smaller the standard deviation, the more stable the flow of traffic will be, and the fewer breakdowns to be expected at high traffic volumes.

Especially when autonomous vehicles are able to anticipate the actions of preceding vehicles through communication, they can contribute to a stabilization of traffic flow and thus to stability. In purely autonomous traffic, it is to be assumed that full stability will be achieved and a capacity drop avoided.

\subsubsection{Intersections with Traffic Lights}

Since intersections as part of streets with high traffic are usually controlled using traffic signals, the following considerations relate to the capacity of intersections with traffic signal control.

At high traffic loads at intersections with traffic lights, constant queues of traffic occur independently of the coordination of the traffic signals. This is why it is usually the case that the waiting queue of vehicles, once permitted by the green light to move, starts moving from standstill. When the first vehicle has departed at a green light, the next follows once a certain time interval has lapsed. This time interval is represented by the value of the time variable, which for standard conditions (no slope, traffic travels in a straight line, lane width is adequate) and pure passenger car traffic amounts to $t_{b}=1.8 \mathrm{~s}$ [10]. This corresponds to a saturation flow of $q_{\mathrm{s}}=2000 \mathrm{cars} / \mathrm{h}$. The value for trucks and buses is $t_{b}=3.15 \mathrm{~s}$, and $t_{b}=4.5 \mathrm{~s}$ for semi-trailer truck.

At the start of the column of traffic, the time at which departure occurs can be anticipated from the movement of vehicles ahead. Thus the response time to the departure of the driver directly in front is reduced and can be assumed to be $T_{h}=0.6 \mathrm{~s}$. At a vehicle length of $4.5 \mathrm{~m}$ and a distance of $3.0 \mathrm{~m}$ from bumper to bumper, an average footprint of a car in the traffic backlog in front of a traffic light may be assumed to be $7.5 \mathrm{~m}$, and plausible values for the average speed at the stop line of the traffic signal to be $v=22.5 \mathrm{~km} / \mathrm{h}$. One can thus confirm the required time interval via the relation $t_{b}=T_{h}+L / v=1.8 \mathrm{~s}$. This applies equally to the amount of time required by trucks and semi-trailer trucks with lengths of 12 and $18 \mathrm{~m}$ respectively. Consequently, the saturation flow of a lane at a traffic signal can be given by the equation of state:

$$
q_{\mathrm{s}}=\frac{v}{v T_{h}+L} \text {. }
$$


To analyze the saturation volumes of purely autonomous and mixed traffic, the correlations used here are those derived for the capacity of road sections with assumed values for queues starting at traffic signals of $22.5 \mathrm{~km} / \mathrm{h}=6.25 \mathrm{~m} / \mathrm{s}, T_{h}=0.6 \mathrm{~s}$. For autonomous driving, it is assumed that the reaction time or the safety margin, even in dense and slow urban traffic, should not fall below $T_{a}=0.3 \mathrm{~s}, T_{a a}=0.3 \mathrm{~s}, T_{a h}=0.6 \mathrm{~s}, T_{h x}=0.6 \mathrm{~s}$.

The capacity of a lane at intersections with traffic lights is, on the one hand, determined by saturation traffic volumes and, on the other hand, by green intervals. Green intervals that are allocated to different traffic streams over the period of one hour are themselves affected by the cycle times and the clearance intervals. During rush hours, a cycle time of $90 \mathrm{~s}$ is usually chosen, meaning that there are forty clearance intervals within an hour. For a typical road junction situated on an urban main street, a three-phase signal program is normally used. The clearance intervals used for the main traffic direction in the three phase-transitions typically add up to around $20 \mathrm{~s}$ and are essentially dependent on the clearance times of crossing pedestrians. With a cycle time of $90,70 \mathrm{~s}$ remain for the green light intervals of the various traffic flows. If one assumes that, from the remaining green interval, $50 \%$ of the time is available for the traffic flows in the main direction, within one hour a release time of $1400 \mathrm{~s}$ and a release time share of $p_{F}=38.89 \%$ results, i.e., a share of approximately $40 \%$.

In conflict-free signaling (i.e., there are no conditional compatibilities, e.g., with pedestrians crossing parallel to the main traffic), the capacity of mixed traffic flows is determined using the above approach.

$$
C_{\mathrm{LSA}}=q_{\mathrm{s}} \cdot p_{F}=\frac{v \cdot p_{F}}{v T_{h}+L}
$$

In current traffic conditions where vehicles are exclusively controlled by humans, when using the above values a capacity of about $800 \mathrm{cars} / \mathrm{h}$ per lane results. In purely autonomous traffic with $T_{a}=0.3 \mathrm{~s}$, the capacity would increase to about $1120 \mathrm{cars} / \mathrm{h}$ and thus increase by about $40 \%$. For a mixed composition of traffic flows, the efficiency gains are between these stated values and can be determined with the formulas introduced above.

The formula also makes it clear that, in addition to the duration of the time delays of following cars, it is especially speed that determines capacity (Fig. 16.11). With increasing clearance speeds, capacity grows with autonomous traffic at a higher rate than that of traffic with human drivers. If it is possible, therefore, to achieve faster departure times and clearance with autonomous driving, as well as shorter time delays, then a significantly higher capacity gain than the $40 \%$ named above can be expected.

\subsubsection{Assessing the Efficiency Gains from Autonomous Driving}

Estimates of the effect of autonomous vehicles on capacity as a measure of the efficiency of transport systems show significant potential for increasing traffic flow, both on some sections of highways and intersections of major urban roads. 
Fig. 16.11 Capacities for a single lane at a traffic signal in relation to clearance speed

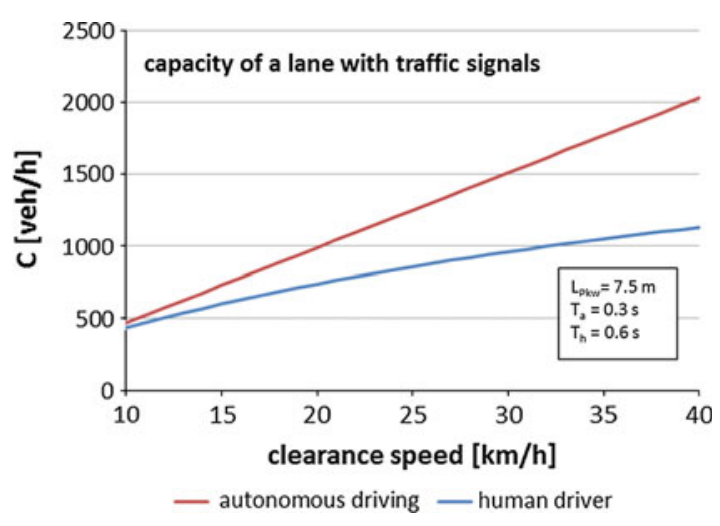

In city traffic, a capacity increase of about $40 \%$ could be achieved with purely autonomous traffic, while capacities could be increased on highway sections by about $80 \%$. The significant difference in the growth potential is due to the average speed at which vehicles drive when using the traffic infrastructure. This is clear from Fig. 16.7, which shows a disproportionate increase in capacity in the range of lower speeds and a flattening-out towards higher speeds. When capacity is reached, the speeds on highways are about $80 \mathrm{~km} / \mathrm{h}$. On urban main roads, the platoon starting at green, which determines capacity at signal lights, moves at an average of $20 \mathrm{~km} / \mathrm{h}$. Because of this difference in speeds, autonomous vehicles have a very different impact on the capacity of transportation infrastructure elements.

In addition to the capacity level achievable by including autonomous vehicles, the stability of the traffic flow at high traffic volumes is important. In city traffic at a capacity utilization of 70-80\%, there is admittedly a constant traffic backlog before the relevant traffic signal, which is why it is no longer possible to drive through without stopping (green wave) at high traffic loads. Nevertheless, there will be a drop in capacity, as is the case in extra-urban traffic, especially on highways. To this extent, the stability of traffic flow is not compromised on the urban road network until it reaches full capacity-and only then when an overload causes blockages at the intersections.

\subsection{Conclusion and Outlook}

\subsubsection{Traffic}

This analysis, with the help of the macroscopic traffic flow models, shows that, in principle, a significant increase in capacity can be expected from using autonomous vehicles and that this would also enable a more efficient use of the existing transport infrastructure. Along with the expected increase in capacity for existing traffic infrastructure, traffic jams and lost time are reduced, which in turn improve the quality of traffic flow. In particular, two factors are responsible for the increase in capacity: 
(a) One factor is the shortening of headways between autonomous vehicles. In this context, it is significant that ride comfort is maintained, despite the short time gaps, by anticipating the actions of the preceding vehicles and thereby enabling lower acceleration or deceleration. This could also be important for column stability. The intercommunication of vehicles and infrastructure appears to be an important prerequisite for this.

(b) In addition to the duration of the time gap, the speed of the vehicle group is very important. The higher the speed at a constant density, the higher the traffic volume over a cross section. However, achieving high speeds while maintaining traffic density is possible only in purely autonomous traffic. A single human-driven vehicle in the column would lead to slower speeds and reduce the capacity gain.

\subsubsection{Infrastructure}

The models developed for traffic flow and capacity, assuming a given share of autonomous vehicles, show that capacity increases disproportionately highly as the share of autonomous vehicles increases. It should be noted that the shortening of the time gaps comes into effect as early as the first autonomous vehicle; the speed increase at high densities, however, will only be possible for purely autonomous traffic. The introduction of autonomous vehicles will succeed, in the opinion of the author, only in their ability to move safely in mixed traffic, as reserved transit areas would not be socially or economically acceptable, particularly with a low share of autonomous traffic.

However, once a sufficient number of vehicles with autonomous capabilities are participating in traffic, it will be very beneficial to the transport efficiency to create reserved lanes for autonomous driving. The benefits of autonomous vehicles can be maximized by separation due to the nonlinear course of the capacity once nonautonomous vehicles are added to autonomous traffic. In conjunction with specially dedicated lanes, the column speed could also be increased even when traffic demand is higher, which would lead to further significant capacity gains. This is not possible in mixed traffic, since even in traffic with only a few human-driven vehicles, these would dictate the speed.

In an initial analysis of this far-reaching subject, this article has solely focused on the traffic effects of autonomous vehicles on sections of motorways and, with an eye to urban traffic, at intersections with traffic lights. These two driving situations to a large extent determine the quality of the traffic flow. However, there are a number of other relevant driving situations that may have a significant influence on the capacity of the overall system:

(a) Outside of urban areas, these are the entry, merging and exit maneuvers at the intersections of major roads. Firstly, we can look forward here to further developing already-emerging technical solutions with assistance functions, such as the merging assistant, particularly in regard to the possibilities of machine cooperation. Secondly, solutions for structural and regulatory adjustments to transportation facilities are still to be developed. For example, one interesting scenario is where autonomous traffic is 
directed onto separate lanes between motorway intersections. This separation is then removed in intersection areas. In an intersection area, autonomous and human-controlled vehicles thus drive in all lanes and each may perform all maneuvers (autonomous, highly assisted or human-driven) at a perhaps predetermined low speed.

(b) Within urban areas, there are still issues regarding the impact of the so-called conditional compatibility that have to be clarified. Conditional compatibility occurs when different traffic flows crossing at traffic signals are given the green light, requiring rules of right of way to be adhered to. This, for example, is the case in traffic flows turning right or left, which must grant right of way to pedestrians and cyclists travelling parallel to the traffic. Various approaches could be interesting for this purpose and should be subjected to closer scrutiny. Thus, one could give the green light to all lanes of autonomous vehicles simultaneously in a separate phase - the maneuvers of the conflicting flows in the intersection area would be negotiated independently by the autonomous vehicles. All other road users would be controlled by the existing signaling. Another possible solution would be to consider cyclists and pedestrians in a separate phase with "all green", thus maximizing vehicle-flow compatibility by using an appropriate phase structure.

\subsubsection{Cooperation}

For scenarios such as this last, where traffic at intersections is self-organizing, autonomous vehicles need to be able to communicate among themselves and with the infrastructure. Anticipating the maneuvers of moving vehicles ahead, and the reactions in the next column that depend on this, results in a comfortable and thus acceptable rate of acceleration, also ensuring the experience of travelling in the vehicle is pleasant. For this reason, currently existing technologies for communication and cooperation will play an important role in the development of autonomous driving.

Open Access This chapter is distributed under the terms of the Creative Commons Attribution 4.0 International License (http://creativecommons.org/licenses/by/4.0/), which permits use, duplication, adaptation, distribution and reproduction in any medium or format, as long as you give appropriate credit to the original author(s) and the source, a link is provided to the Creative Commons license and any changes made are indicated.

The images or other third party material in this chapter are included in the work's Creative Commons license, unless indicated otherwise in the credit line; if such material is not included in the work's Creative Commons license and the respective action is not permitted by statutory regulation, users will need to obtain permission from the license holder to duplicate, adapt or reproduce the material.

\section{References}

1. Greenshields, B.D. (1935) A study of highway capacity. Proceedings Highway Research Record, (Washington) 14, 448-477

2. May, A.D., Keller, H. (1968) Evaluation of single- and two-regime traffic flow models. Straßenbau und Straßenverkehrstechnik 86, 37-47 
3. FGSV - Forschungsgesellschaft für Straßen- und Verkehrswesen (2001) Handbuch für die Bemessung von Straßenverkehrsanlagen (HBS), pp. 3-19

4. Janiszewski, H., Jagow, J., Burmann, M. (2005) Straßenverkehrsrecht. Beck Juristischer Verlag, Munich

5. Wagner, P. (2014 a) Traffic control and traffic management in a transportation system with autonomous vehicles. Present volume (Chap. 15)

6. Brilon, W., Regler, M., Geistefeldt, J. (2005) Zufallscharakter der Kapazität von Autobahnen und praktische Konsequenzen. Straßenverkehrstechnik 3(1) and 4(2)

7. Friedrich, B., Kemper, C. (2006) Akzeptanz von Harmonisierungsmaßnahmen im Zuge von Verkehrsbeeinflussungsanlagen. Schlussbericht für die Niedersächsische Landesbehörde für Straßenbau und Verkehr, Hannover

8. Hall, F.L., Agyemang-Duah, K. (1991) Freeway capacity drop and the definition of capacity. Transportation Research Record 1320, TRB, National Research Council, Wash. D.C.

9. Wagner, P. (2014 b) private correspondence

10. FGSV - Forschungsgesellschaft für Straßen- und Verkehrswesen (2014) Handbuch für die Bemessung von Straßenverkehrsanlagen (HBS) - Entwurf der Neufassung 